\title{
ADRC, una estrategia de control robusto
}

\section{ADRC, a robust control strategy}

\author{
Carlos Vergara Ramírez' \\ Nelson Castañeda Arias² \\ Álvaro Cortés Prieto ${ }^{3}$
}

\section{Resumen}

Este artículo pretende ilustrar la estrategia conocida como Control por Rechazo Activo de Perturbaciones (ADRC por sus siglas en inglés), por medio del problema de seguimiento de posición de una esfera metálica en un levitador magnético. Este enfoque de control permite realizar estimaciones por medio de observadores lineales de dinámicas no modeladas, reduciendo así la complejidad de la ley de control y proporcionando una robustez considerable al sistema. Se realizó la validación de la ley de control por simulación obteniendo excelentes resultados en el seguimiento de trayectorias suaves, aun en presencia de incertidumbres paramétricas del modelo y perturbaciones externas.

Palabras clave: control robusto, levitación magnética, observadores lineales, rechazo a perturbaciones.

\section{Abstract}

This paper tries to illustrate the strategy known as Active Disturbance Rejection Control, ADRC, by means of a tracking position of a metallic sphere in a magnetic levitator. This control approach allows to make some estimations by means of linear observers of non-modified dynamics reducing in that way the complexity of the control law and providing a considerable robustness to the system. A validation of the control law was performed by simulation obtaining excellent results in the tracking of smooth trajectories even in the presence of parametric model uncertainties and external perturbations.

Keywords: Robust control, Magnetic levitation, Lineal observers, Disturbance rejection.

1 Ingeniero en control, especialista en Informática y Automática Industrial, estudiante de maestría en Automatización Industrial, director del grupo de investigación Techne, docente de las asignaturas de Control, Facultad de Mecatrónica. Correo electrónico: cfvergarar@itc.edu.co

2 Ingeniero en control, magíster en Automatización Industrial, director del semillero Isqua, docente en las asignaturas de control, Facultad de Mecatrónica. Correo electrónico: nacastanedaa@itc.edu.co

3 Ingeniero electricista, investigador del grupo Techne, docente de las asignaturas de circuitos y proyectos de la Facultad de Mecatrónica. Correo electrónico: aprieto@itc.edu.co 


\section{Introducción}

El levitador magnético simple es una planta típicamente usada para la enseñanza de los sistemas de control, debido a su dinámica altamente no lineal, naturalmente inestable, con alta incertidumbre paramétrica y fácilmente perturbable (Zhang, Xian y Ma, 2015; Venghi, González y Serra, 2016; Back y Shim, 2014; Assis y Galvao, 2017). Estas características motivaron el diseño de un controlador desde la estrategia de rechazo activo de perturbaciones que solucione el problema de seguimiento de manera robusta. El control propuesto tiene como uno de sus ejes los observadores de tipo lineal para la estimación de incertidumbres, esto con el fin de plantear una ley de control robusta para una amplia clase de sistemas conocidos como diferencialmente planos (Sira y Agrawal, 2004; Sira, Luviano y Cortés, 2011).

Si bien el sistema de levitación magnética es un problema muy académico, permite tener un acercamiento al control de sistemas eléctricos con cargas inductivas, lo cual es muy recurrente en los distintos tipos de máquinas de la industria. Controladores que toleran pequeñas incertidumbres implementados sobre cargas inductivas suelen tener dificultades para seguir referencias, e incluso estabilizar la planta debido a la variación en la impedancia, alteración del campo magnético, presencia de armónicos, entre otros. Por otra parte, la clase compensadores propuestos suelen ser de tipo no-lineal, por lo que la implementación puede llegar a ser compleja.

Una propuesta de solución clásica a los problemas de control de sistemas con no-linealidades es linealizar, lo que permite implementar controladores ampliamente conocidos, como los PID (Rairán y Millán, 2002), la dificultad radica en que los procesos de linealización limitan la zona de trabajo y la robustez del sistema. Otro inconveniente, que en general poseen todos los sistemas de control clásico, es el debido al empobrecimiento del desempeño ante perturbaciones externas.
Todo esto ha motivado el diseño de una estrategia de control que dote con una robustez al sistema realimentado que permita tolerar incertidumbres y perturbaciones, en una zona de trabajo amplia, siguiendo distintos tipos de referencias suaves y que su implementación no resulte compleja.

\section{Marco teórico}

\subsection{Modelo}

Como base para el diseño del controlador y la simulación del sistema que se muestra en la figura 1 , se deducen las ecuaciones que representan la dinámica del sistema, analizando los dos fenómenos físicos existentes en la planta, mecánico y electromagnético, tal y como lo presenta Khalil (2002).

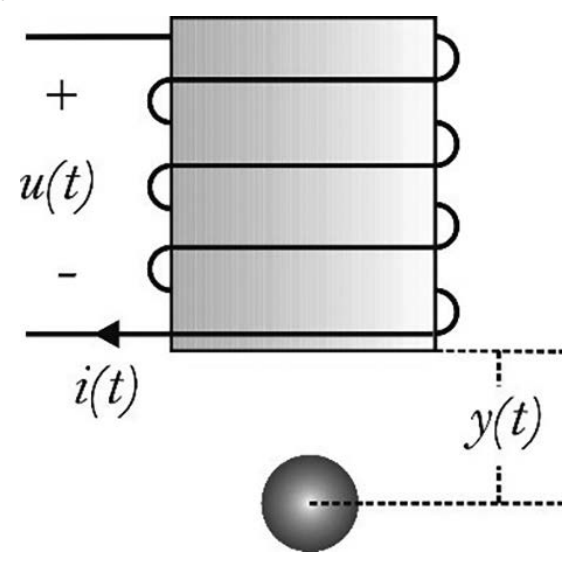

Figura 1. Sistema de levitación magnética simple Fuente autores

El fenómeno mecánico se puede describir con ayuda de la Ley de Newton, como se muestra en la ecuación 1, donde es la distancia desde el electroimán hasta la esfera, y y $\ddot{y}$ son la primera y segunda derivada de respecto al tiempo respectivamente, la masa de la esfera, el coeficiente de fricción viscosa y $\mathrm{F}(\mathrm{y}, \mathrm{i})$ la fuerza debida al campo magnético inducido por el electroimán que depende además de la corriente $\mathrm{i}$.

$$
\mathrm{m} \ddot{y}=-\mathrm{k} \dot{y}+\mathrm{mg}+\mathrm{F}(y, i)
$$

El componente eléctrico del sistema es posible modelarlo con ayuda de la ley de voltaje de Kir- 
chhoff, como se muestra en la ecuación 2, siendo $\mathrm{R}$ la resistencia del devanado, $\phi$ el flujo magnético y $\mathrm{u}(\mathrm{t})$ el potencial eléctrico y para este caso la señal de control.

$$
u(t)=\phi^{\prime}(t)+\operatorname{Ri}(t)
$$

El flujo magnético se puede relacionar con la corriente de la siguiente manera:

$$
\phi=\mathrm{L}(\mathrm{y}) \mathrm{i}
$$

Siendo L(y) la inductancia de devanado que varía según la posición de la esfera, como se muestra a continuación:

$$
L(y)=L_{1}+\frac{L_{0}}{1+\frac{y}{a}}
$$

Por último, la conversión electromagnética es debido al almacenamiento de la energía eléctrica en la inductancia $\mathrm{E}=\frac{1}{2} \mathrm{~L}(\mathrm{y}) \mathrm{i}^{2} \quad$ y por definición la fuerza producida por esta energía se presenta en la siguiente relación.

$$
F(y, i)=\frac{\partial E}{\partial y}=-\frac{L_{0} i^{2}}{2 a\left(1+\frac{y}{a}\right)^{2}}
$$

Con ayuda de las ecuaciones 1, 2 y demás relaciones anteriormente presentadas, es posible realizar una representación en espacio de estados del sistema, como se muestra en la ecuación 3 , siendo $x_{1}=y, x_{2}=\dot{y} y x_{3}=i$.

$$
\begin{aligned}
& \dot{x}_{1}=x_{2} \\
& \dot{x}_{2}=g-\frac{k}{m} x_{2}-\frac{L_{0} a x_{3}^{2}}{2 m\left(a+x_{1}\right)^{2}} \\
& \dot{x}_{3}=\frac{1}{L\left(x_{1}\right)}\left[-R x_{3}+\frac{L_{0} a x_{2} x_{3}}{\left(a+x_{1}\right)^{2}}+u(t)\right]
\end{aligned}
$$

\subsection{Sistemas diferencialmente planos}

Los sistemas diferencialmente planos hacen parte de una clase muy frecuente de sistemas lineales y no lineales, con la intención de revisar su principal característica suponga un sistema SISO (una salida y una entrada) cualesquiera descrito por la ecuación 4, donde son las variables de estado y la entrada del sistema (Sira y Agrawal, 2004).

$$
x^{\prime}=f(x, u), x \in R^{n}, u
$$

Entonces se dice que el sistema anterior es diferencialmente plano si todas las variables de estado se pueden definir en términos de las salidas y un número finito de sus derivadas en función del tiempo, además si se puede establecer una relación entrada salida como la que se muestra a continuación (Sira et al., 2011).

$$
y^{(n)}=\kappa(x) u(t)+\psi(x)
$$

Donde $\mathrm{y}^{(\mathrm{n})}$ representa la derivada temporal de la salida.

\subsection{Rechazo activo de perturbaciones.}

En este artículo se diseñó un control basado en el paradigma de rechazo activo de perturbaciones (Gao, 2006) y observadores de tipo Proporcional Integral Generalizado (GPI) (Fuentes, Romero y Triana, 2014). El método es directamente aplicable a sistemas diferencialmente planos planteando una ley de control desde la relación entrada salida, en donde lo fundamental a conocer del sistema es el grado relativo (n) y la función que pondera la entrada $(\kappa(\mathrm{x})$, la robustez del sistema de control está basada en la estimación de perturbaciones de tipo aditivo, con la ayuda de un observador lineal clásico extendido.

Con la finalidad de ilustrar el procedimiento usado, suponga el sistema mostrado en la ecuación 6 , donde la variable $\zeta(\mathrm{t})$ representa las perturbaciones externas o incertidumbres del modelo.

$$
y^{(n)}=\kappa(x) u(t)+\psi(x)+\zeta(t)
$$

Es posible reescribir lo anterior, ecuación 7, en donde $\xi(t)$ se define como la perturbación generalizada, de tipo aditivo. 


$$
\mathrm{y}^{(\mathrm{n})}=\kappa(\mathrm{x}) \mathrm{u}(\mathrm{t})+\xi(\mathrm{t})
$$

Una ley de control que puede llevar la r-esima derivada del error de seguimiento $\left(e^{(r)}\right)$ a cero de manera asintótica y exponencial, con $\mathrm{e}^{(\mathrm{r})}=\mathrm{y}^{(\mathrm{r})}$ $\left[y^{*}\right]^{(r)}$ y $r=0,1, \ldots, n$, sería (Sira et al., 2011).

$$
\begin{aligned}
& u(t)=\kappa^{-1}(x)\left(\left[y^{*}\right]^{(n)}-\sum_{r=0}^{n-1} \alpha_{r} e^{(r)}-\hat{\xi}(t)\right) 8 \\
& u(t)=\kappa^{-1}(x)(v-\hat{\xi}(t))
\end{aligned}
$$

Donde $\xi^{\wedge}(x)$ es la estimación de la perturbación generalizada, y $\alpha_{r}$ son los coeficientes que determinan la dinámica del error de seguimiento:

$$
\mathrm{e}^{(\mathrm{n})}+\alpha_{\mathrm{n}-1} \mathrm{e}^{(\mathrm{n}-1)}+\cdots+\alpha_{1} \mathrm{e}^{\cdot}+\alpha_{0} \mathrm{e}=0
$$

Por último, la perturbación generalizada se estima con ayuda de un observador de estados extendido, suponiendo que es posible modelarla por medio de un polinomio en función del tiempo (Sira et al., 2011):

$$
\xi(\mathrm{t})=\mathrm{k}_{0}+\mathrm{k}_{1} \mathrm{t}+\mathrm{k}_{2} \mathrm{t}^{2}+\cdots+\mathrm{k}_{\mathrm{m}} \mathrm{t}^{\mathrm{m}}+\mathrm{r}(\mathrm{t})
$$

Con $r(t)$ el residuo, si se asume el modelo para la estimación de la perturbación, como $\hat{\xi}^{(\mathrm{m})}(\mathrm{t})=0$ es posible plantear un observador para las derivadas de la salida, la perturbación y sus derivadas (Fuentes et al., 2014):

$$
\begin{aligned}
& \dot{y}_{0}=y_{1}+\lambda_{(n+m-1)}(y-\hat{y}) \\
& \dot{y}_{0}=y_{2}+\lambda_{(n+m-2)}(y-\hat{y}) \\
& \begin{array}{ccll}
\vdots & \vdots & \vdots \\
\dot{y}_{(n-1)} & = & \kappa(\hat{x}) u(t)+\xi_{0}+\lambda_{m}(y-\hat{y}) & 10
\end{array} \\
& \dot{\xi}_{0}=\xi_{1}+\lambda_{(m-1)}(y-\hat{y}) \\
& \dot{\xi}_{1}=\xi_{2}+\lambda_{(m-2)}(y-\hat{y}) \\
& \vdots \quad \vdots \quad \vdots \\
& \dot{\xi}_{(m-1)}=\lambda_{0}(y-\hat{y})
\end{aligned}
$$

Siendo, $y \mathrm{I}=\mathrm{y}^{(\mathrm{i})}$, la i-esima derivada temporal de la estimación de la salida plana y, y $\xi_{\mathrm{i}}=\xi^{(\mathrm{j})}$ la j-esima y la derivada temporal de la estimación de la perturbación generalizada $\xi$.

\section{Metodología}

A continuación, se describe el procedimiento para el diseño del control del levitador magnético.

\subsection{Planitud diferencial del sistema}

Dado que el problema del control está enfocado a la posición de la esfera, se supone el acceso a la medición de esta variable, por lo tanto, la salida plana del sistema sería $\mathrm{x}_{1}$. En la ecuación \ ref\{equ:PlanitudSistema\} se definen las variables de estado en términos de la salida plana y sus derivadas.

$\begin{aligned} x_{2} & =\dot{x}_{1} \\ x_{3} & =\left[\sqrt{\left(-\ddot{x}_{1}+g-\frac{k}{m} \dot{x}_{1}\right) \frac{2 m}{a L_{0}}}\right]\left(a+x_{1}\right)\end{aligned}$

En la siguiente ecuación es posible ver que la relación entrada salida se encuentra en la tercera deriva temporal de $\mathrm{x}_{1 \text { ", }}$ esto quiere decir que el sistema es de grado relativo tres y es diferencialmente plano.

$$
x_{1}^{(3)}=\frac{-k}{m} \dot{x}_{2}-\frac{a L_{0}\left[\left(a+x_{1}\right)^{2} x_{3 x_{3}}+x_{3}^{2}\left(a+x_{1}\right) \dot{x}_{1}\right]}{m\left(a+x_{1}\right)^{4}}
$$

Como resultado de esto es posible representar nuestro sistema como en la ecuación 7, en donde:

$$
\kappa(x)=\frac{-\mathrm{aL}_{0} \mathrm{x}_{3}}{\mathrm{~L}\left(\mathrm{x}_{1}\right)\left(\mathrm{a}+\mathrm{x}_{1}\right)^{2}}
$$

Y

$$
\xi(\mathrm{t})=\frac{\mathrm{L}_{0} \mathrm{ax}_{3}}{\mathrm{~m}\left(\mathrm{a}+\mathrm{x}_{1}\right)^{2}}\left(\frac{-\mathrm{Rx}_{3}}{\mathrm{~L}\left(\mathrm{x}_{1}\right)}+\frac{\mathrm{L}_{0} \mathrm{x}_{2} \mathrm{x}_{3}}{\mathrm{~L}\left(\mathrm{x}_{1}\right)\left(\mathrm{a}+\mathrm{x}_{1}\right)^{2}}\right)+\frac{\mathrm{L}_{0} \mathrm{ax}_{3} \dot{x}_{1}}{\mathrm{~m}\left(\mathrm{a}+\mathrm{x}_{1}\right)^{3}}-\frac{\mathrm{k}}{\mathrm{m}} \dot{x}_{2}
$$

\subsection{Ley de control}

La ecuación 8 representa una ley de control que puede llevar el error de seguimiento a cero de manera asintótica y exponencial a cero, sin embargo, la única variable medible para este sistema es $\mathrm{x}_{1}$, las otras variables de estado será necesario estimarlas, de esto resulta que la función $\kappa(x)$ 
tendrá algunas variables de estado estimadas, y en adelante se notará como $\mathrm{k}(\hat{\mathrm{x}})$, quedando para este sistema como sigue.

$$
\kappa(\hat{x})=\frac{-a L_{0} \hat{x}_{3}}{L\left(x_{1}\right)\left(a+x_{1}\right)^{2}}
$$

Es importante resaltar que las variables necesarias para formar la ley de control que no sean medibles se estimarán por medio del observador GPI propuesto en la ecuación 10, y las relaciones debidas a la planitud diferencial descritas en la ecuación 11. La ley de control auxiliar para este caso estaría descrita como se muestra en la siguiente ecuación.

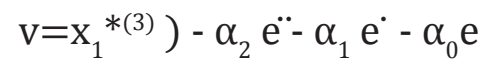

Para seleccionar los respectivos valores de los coeficientes se hace uso de la sintonización a partir de la minimización de la integral del error absoluto ITAE, como lo presenta Chen (1995), resultando los siguientes valores:

$\alpha_{2}=617.75 ; \alpha_{1}=404.97 * 10^{3} ; \alpha_{0}=43.98 * 10^{6}$

\subsection{Diseño del observador}

De acuerdo con la ley de control planteada, es necesario estimar las variables $\mathrm{x}_{3}, \mathrm{x}_{2}, \mathrm{x}_{1} \mathrm{y} \xi$. Según la ecuación 10, es posible plantear el observador GPI para este sistema:

$$
\begin{array}{lcc}
\dot{y}_{0}= & y_{1}+\lambda_{5}(y-\hat{y}) \\
\dot{y}_{1}= & y_{1}+\lambda_{4}(y-\hat{y}) \\
\dot{y}_{2}= & \kappa(\hat{x}) u+\xi_{0}+\lambda_{3}(y-\hat{y}) \\
\dot{\xi}_{0}= & \xi_{1}+\lambda_{2}(y-\hat{y}) \\
\dot{\xi}_{1}= & \xi_{2}+\lambda_{1}(y-\hat{y}) \\
\dot{\xi}_{2}= & \lambda_{0}(y-\hat{y})
\end{array}
$$

La ecuación anterior implica que el modelo seleccionado de la perturbación generalizada corresponde $\left.a \cdot \xi^{(3)}\right)(\mathrm{t})=0$. Por último, se seleccionan las constantes $\lambda \mathrm{i}$, de tal forma que la dinámica del observador sea al menos tres veces más rápida que la del error, para este caso los valores de los coeficientes son:

$$
\begin{gathered}
\lambda_{5}=6.053 * 10^{3} ; \lambda_{4}=13.12 * 10^{6} ; \lambda_{3}=16.59 * 10^{9} \\
\lambda_{2}=13.52 * 10^{12} ; \lambda_{1}=6.39 * 10^{15} ; \lambda_{0}=935.788 * 10^{15}
\end{gathered}
$$

Como resultado se crea la ley de control (figura 2), donde u es la ley de control, Obs el observador de estados extendidos, y $(\mathrm{t}) *$ la salida de la planta y la señal de referencia.

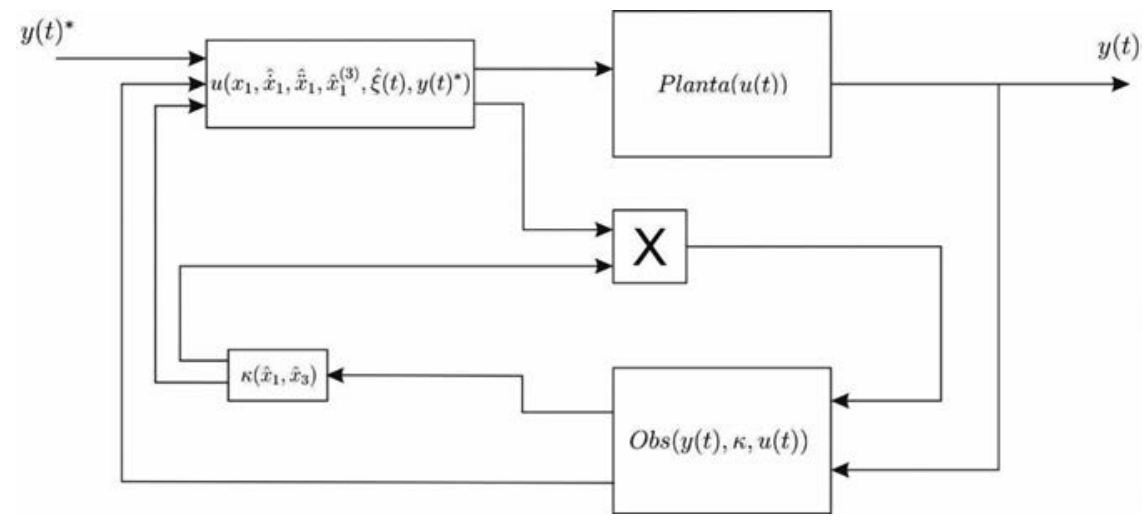

Figura 2. Arquitectura de control propuesta.

Fuente autores

\section{Resultados y discusión}

Se llevó a cabo la simulación del sistema en Matlab-Simulink, donde se realizaron distintos escenarios para comprobar el funcionamiento del sistema de control.

\subsection{Seguimiento de señales de referencia}

Se diseñó una señal de referencia de quinto orden y se aplicó al sistema, su respuesta puede verse a continuación. 


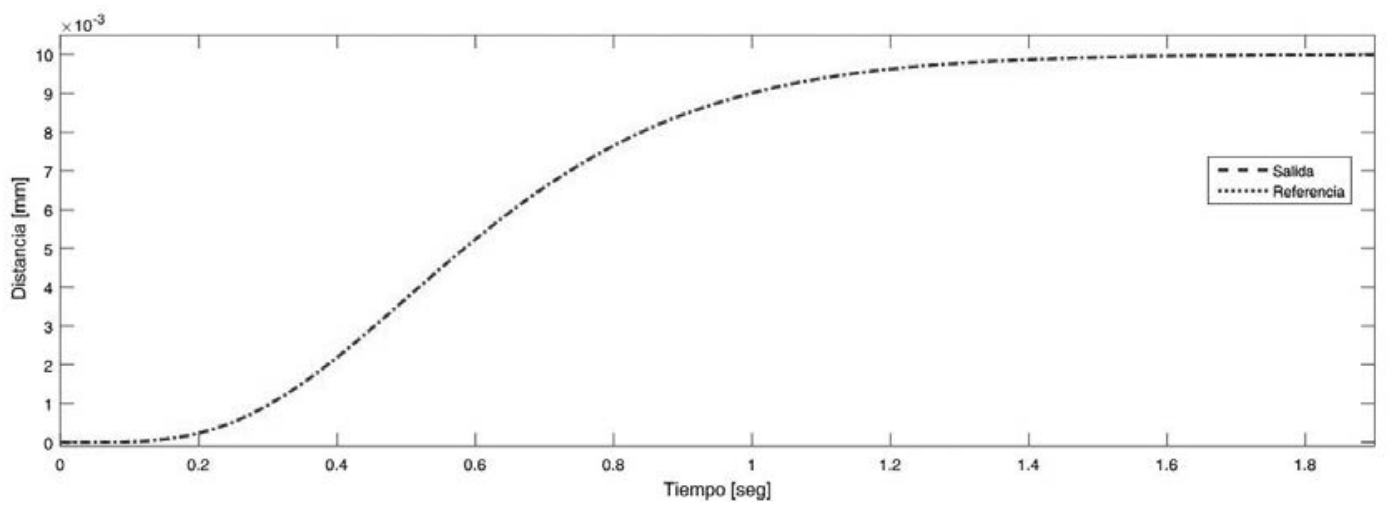

Figura 3. Respuesta del sistema a cierta referencia Fuente. autores

Luego se aplicó una señal sinusoidal de amplitud y una frecuencia de $10 \mathrm{rad} / \mathrm{s}$ (figura 4), logrando un error absoluto de $7.76 \mu \mathrm{m}$ :

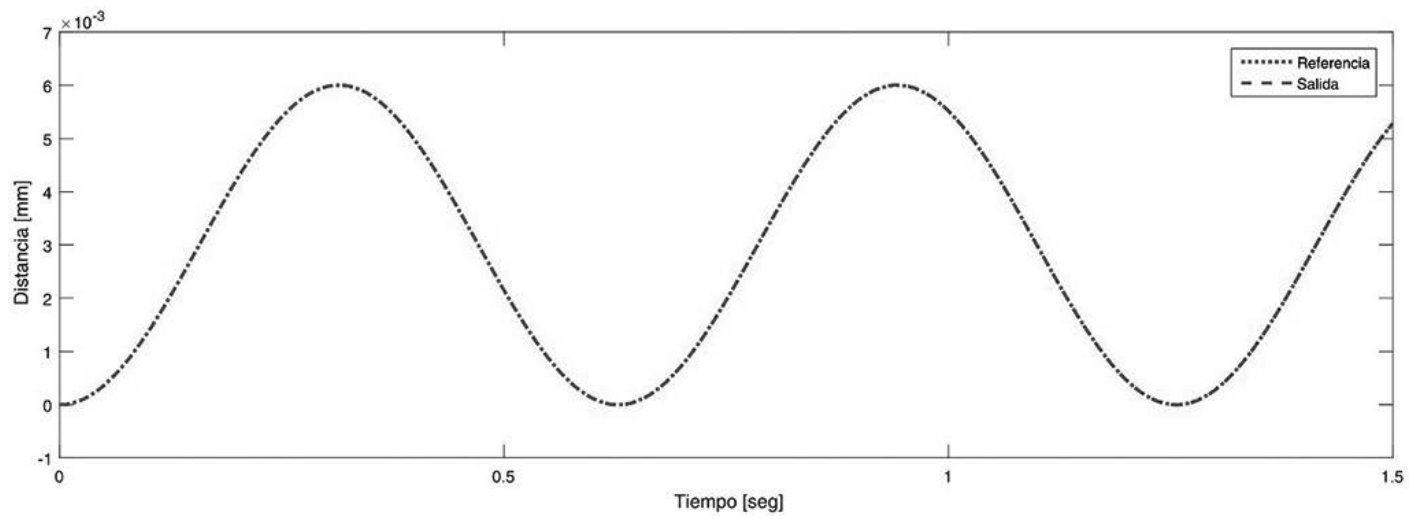

Figura 4. Respuesta del sistema a referencia senoidal.

Fuente autores

\subsection{Estimación de estados}

Se compara la salida del estimador de estados con las salidas correspondientes de la planta con una señal de referencia sinusoidal, como la presentada en la sección anterior. Obteniendo los siguientes resultados:

Error absoluto de estimación en el estado $x_{1}$ fue de: $176 \mathrm{pm}$.

Error absoluto de estimación en el estado $x_{2}$ fue de: $48 \mathrm{~mm} / \mathrm{s}$.

Error absoluto de estimación en el estado $x_{3}$ fue de: $290 \mu$ A.

\subsection{Cambios paramétricos de la planta}

Se modificaron los parámetros del sistema obteniendo robustez en el desempeño hasta con cambios en los parámetros del 30\%, obteniendo un error absoluto máximo de: $5.37 \mu \mathrm{m}$.

\subsection{Perturbaciones externas}

Se aplicó una perturbación externa tipo impulso en el segundo con una magnitud equivalente al del peso de la esfera, y se obtuvo un error absoluto de: $3.19 \mu \mathrm{m}$., la respuesta se evidencia en la figura 5 y 6. 


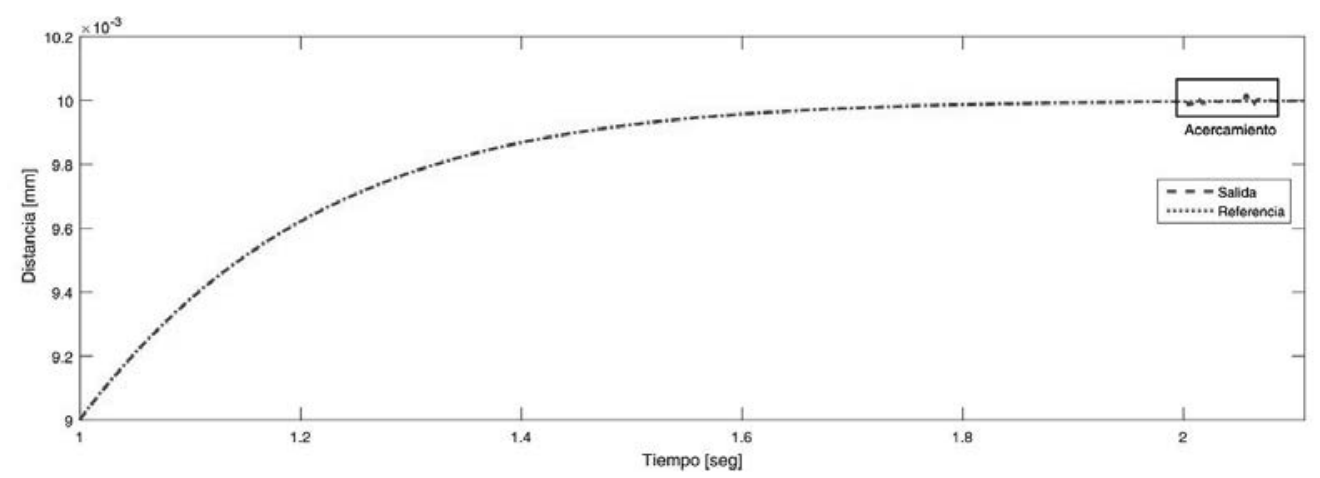

Figura 5. Respuesta de sistema bajo perturbación externa.

Fuente autores

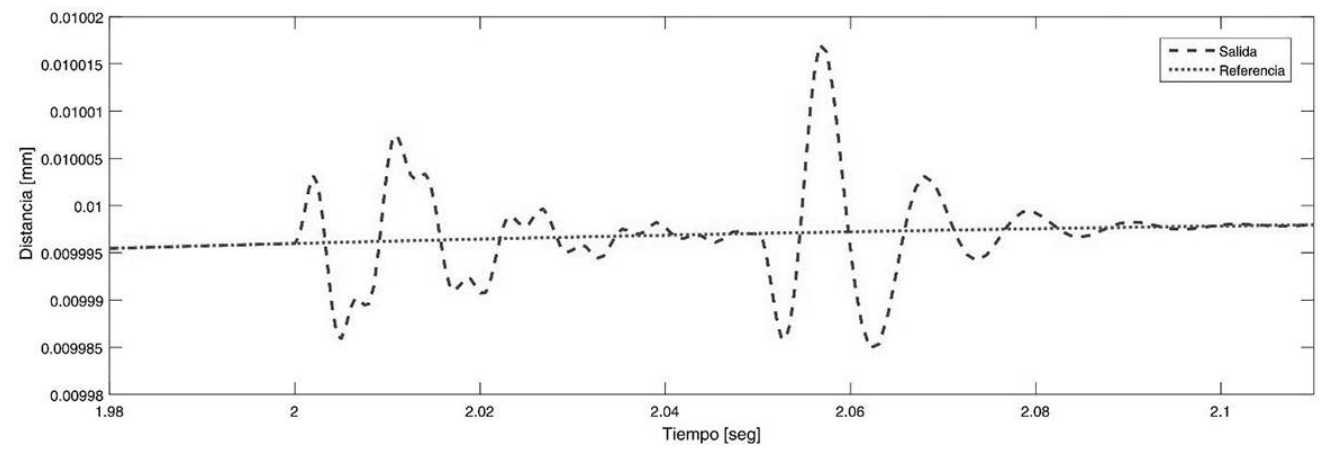

Figura 6. Acercamiento momento de perturbación.

Fuente autores

\section{Conclusiones}

Dada la estructura de la ley de control (ecuación
De acuerdo con los resultados obtenidos, es posible realizar el seguimiento de trayectorias suaves con error inferior al incluso ante incertidumbres paramétricas y perturbaciones externas.

De acuerdo con las simulaciones realizadas, es necesario ajustar las condiciones iniciales de los observadores, de tal forma que sean cercanas a las de la planta, esto debido a que la cadena de integradores propuesta como observadores puede generar señales de control muy grandes, incluso singularidades.

Debido que el desarrollo de la ley de control es similar a la de linealización entrada salida, clásica del control no lineal, es necesario revisar las singularidades que se puedan presentar en dicha ley.

\section{Referencias bibliográficas}

Assis, P. A., y Galvao, R. K. (Febrero de 2017). Sliding mode predictive control of a magnetic levitation system employing multi-parametric programming. IEEE Latin America Transactions, 15(2), 239-248. 
Back, J., y Shim, H. (2014). Reduced-order implementation of disturbance observers for robust tracking of non-linear systems. IET Control Theory Applications, 8(17), 1940-1948.

Chen, C. T. (1995). Analog and Digital Control System Design: Transfer-function, State-space, and Algebraic Methods. New York, NY, USA: Oxford University Press, Inc.

Fuentes, G. R., Romero, J. C., y Triana, A. J. (2014). Control proporcional integral generalizado para señales periódicas. Revista Tecnura, 17, 18-32.

Gao, Z. (Junio de 2006). Active disturbance rejection control: a paradigm shift in feedback control system design. 2006 American Control Conference, 7.

Khalil, H. K. (2002). Nonlinear systems. Prentice-Hall.

Rairán, D., y Millán, F. J. (2002). Levitación magnética de una esfera utilizando un compen- sador PID digital y etapa potencia PWM. Revista Tecnura, 5(10), 34-41.

Sira, R. H., y Agrawal, S. K. (2004). Differentially flat systems. Marcel Dekker Inc.

Sira, R. H., Luviano, J. A., y Cortés, R. J. (2011). Control lineal robusto de sistemas no lineales diferencialmente planos. Revista Iberoamericana de Automática e Informática Industrial RIAI, 8(1), 14-28.

Venghi, L. E., González, G. N., y Serra, F. M. (Junio de 2016). Implementation and Control of a Magnetic Levitation System. IEEE Latin America Transactions, 14(6), 26512656.

Zhang, Y., Xian, B., y Ma, S. (Septiembre de 2015). Continuous Robust Tracking Control for Magnetic Levitation System With Unidirectional Input Constraint. IEEE Transactions on Industrial Electronics, 62(9), 5971-5980. 